\title{
Disintegration of quarkonia in QGP due to time dependent potential
}

\author{
Partha Bagchi* and Ajit M. Srivastava \\ Institute of Physics \\ Bhubaneswar, India, 751005 \\ E-mail: parphy85@gmail.com, ajit@iopb.res.in
}

\begin{abstract}
Rapid thermalization in ultra-relativistic heavy-ion collisions leads to fast changing potential between a heavy quark and antiquark from zero temperature potential to the finite temperature one. Time dependent perturbation theory can then be used to calculate the survival probability of the initial quarkonium state. In view of very short time scales of thermalization at RHIC and LHC energies, we calculate the survival probability of $J / \psi$ and $\Upsilon$ using sudden approximation. Our results show that quarkonium decay may be significant even when temperature of QGP remains low enough so that the conventional quarkonium melting due to Debye screening is ineffective.
\end{abstract}

9th International Workshop on Critical Point and Onset of Deconfinement

17-21 November, 2014

ZiF (Center of Interdisciplinary Research), University of Bielefeld, Germany

\footnotetext{
${ }^{*}$ Speaker.
} 
Suppression of heavy quarkonia as a signal for the quark-gluon plasma phase in relativistic heavy-ion collisions has been investigated intensively since the original proposal by Matsui and Satz [1]. The underlying physical picture of this suppression is that due to deconfinment, potential between $q \bar{q}$ gets Debye screened, resulting in the swelling of quarkonia. If the Debye screening length of the medium is less than the radius of quarkonia, then $q \bar{q}$ may not form bound states, leading to melting of the initial quarkonium. Due to this melting, the yield of quarkonia will be suppressed. This was proposed as a signature of QGP and has been observed experimentally [2]. However, there are other factors too that can lead to the suppression of $J / \psi$ because of which it has not been possible to use $J / \psi$ suppression as a clean signal for QGP.

In the above picture, suppression of quarkonia occurs when the temperature of QGP achieves a certain value, $T_{D}$, so that the Debye screening melts the quarkonium bound state. Thus, if the temperature remains smaller than $T_{D}$, so that Debye screening length remains larger than the quarkonia size, no suppression is expected. This type of picture is consistent with the adiabatic evolution of a quantum state under changing potential. Original quarkonia has a wave function appropriate for zero temperature potential between a $q$ and $\bar{q}$. If the environment of the quarkonium changes to a finite temperature QGP adiabatically, with Debye screened potential, the final state will evolve to the quarkonium state corresponding to the finite temperature potential. If temperature remains below $T_{D}$, quarkonium wave function changes (adiabatically) but it survives as the quarkonium.

We question this assumption of adiabatic evolution for ultra-relativistic heavy-ion collisions, such as at RHIC, and especially at LHC. At such energies, it is possible that thermalization is achieved in a very short time, about $0.25 \mathrm{fm}$ for RHIC and even smaller about $0.1 \mathrm{fm}$ for LHC [3]. Even conservatively, thermalization is achieved within $1 \mathrm{fm}$ as suggested by the elliptic flow measurements [4]. For $J / \psi$ and even for $\Upsilon$, typical time scale of $q \bar{q}$ dynamics will be at least 1-2 $\mathrm{fm}$ from the size of the bound state and the fact that $q \bar{q}$ have non-relativistic velocities. Also, $\Delta E$ between $J / \psi$ and its next excited state $(\chi)$ is about $300 \mathrm{MeV}$ (400 MeV for $\Upsilon$ states), leading to transition time scale $\sim 0.7 \mathrm{fm}(0.5 \mathrm{fm}$ for $\Upsilon)$. Thus the change in the potential between $q$ and $\bar{q}$ occurs in a time scale which is at most of the same order, and likely much shorter than, the typical time scale of the dynamics of the $q \bar{q}$ system, or the time scale of transition between relevant states. The problem, therefore, should be treated in terms of a time dependent perturbation and survival probability of quarkonia should be calculated under this perturbation. It is immediately clear that even if the final temperature remains less than $T_{D}$, if the change in potential is fast enough invalidating the adiabatic assumption, then transition of initial quarkonium state to other excited states will occur. Such excited states will have much larger size, typically larger than the Debye screening length, and will melt away. Thus quarkonia melting can occur even when QGP temperature remains below $T_{D}$. We mention that adiabatic evolution of quarkonia states has been discussed earlier for the cooling stage of QGP in relativistic heavy ion collisions in the context of sequential suppression of quarkonia states [5]. However, as far as we are aware, validity of adiabatic evolution during the thermalization stage has not been discussed earlier.

Given the large difference between thermalization time scale of order $0.1-0.2 \mathrm{fm}$ [3], and the time scale of $q \bar{q}$ dynamics in a quarkonium bound state being of order 1-2 fm (or the time scale of transition between relevant states being $0.5-0.7 \mathrm{fm}$ ), it may be reasonable to use the sudden perturbation approximation. The initial wave function of the quarkonium cannot change under this sudden perturbation. Thus, as soon as thermalization is achieved with QGP temperature 
being $T_{0}$ (which may remain less than $T_{D}$ for the quarkonium state under consideration), the initial quarkonium wave function is no longer an energy eigen state of the new Hamiltonian with the $q \bar{q}$ potential corresponding to temperature $T_{0}$. One can find overlap with the new eigen states, giving us the survival probability of the quarkonium as well as the probability of its transition to other excited states.

For calculating the zero temperature wave function of the quarkonium we use the following potential between $q$ and $\bar{q}$.

$$
V(r)=-\frac{\alpha_{s}}{r}+\sigma r
$$

where $\alpha_{s}$ is the strong coupling constant and $\sigma$ is the string tension. For $J / \psi$, we will use charm quark mass $m_{c}=1.28 \mathrm{GeV}, \alpha_{s}=\pi / 12$, and $\sigma=0.16 \mathrm{GeV}^{2}$ [6]. For $\Upsilon$, we use the bottom quark mass $m_{b}=4.67 \mathrm{GeV}$.

For calculating wave functions at finite temperature we use the following potential which incorporates Debye screening [6]

$$
V(r)=-\frac{\alpha_{s}}{r} \exp \left(-\omega_{D} r\right)+\frac{\sigma}{\omega_{D}}\left(1-\exp \left(-\omega_{D} r\right)\right)
$$

where $\omega_{D}=T \sqrt{6 \pi \alpha_{s}}$. We have calculated wave functions for charmonium and bottomonium states at different temperatures with above potentials using Numerov method for solving the Schrödinger equation. We have also used energy minimization technique to get the wave functions for the ground states and the binding energy and the results obtained by both the methods match very well. Fig. 1 shows plots of wave functions for $J / \psi$ at $T=0$ and $200 \mathrm{MeV}$. With finite temperature potential (Eq.(2)), excited states of charmonium are not found for $T \geq 200 \mathrm{MeV}$. Fig. 2 shows wave functions for $\Upsilon$ states at $T=0,200,400$, and $500 \mathrm{MeV}$. For Bottomonium, we find excited state $\Upsilon(2 S)$ at $T=200 \mathrm{MeV}$ which is shown in Fig.3, along with the ground state $\Upsilon(1 S)$ at $T=0$.

As we mentioned, we use the sudden approximation to calculate the survival probability of quarkonium state which is calculated directly by calculating (mod square of) the overlap of the wave function of the zero temperature quarkonium state with the wave function of the appropriate state at finite temperature. Figs.1-3 immediately give an idea of this overlap, which is clearly decreasing with increasing temperature implying decreasing survival probability of the quarkonium. Fig. 4 shows the plot of survival probabilities for $J / \psi$ and for $\Upsilon$ as a function of temperature. Survival probabilities are plotted up to a temperature $T_{D}$ beyond which the quarkonium state does not exist any more due to Debye screening in the potential in Eq.(2). We note dramatic decrease in survival probabilities down to about $10 \%$ for both $J / \psi$ and $\Upsilon$ as temperature increases to $270 \mathrm{MeV}$ and $560 \mathrm{MeV}$ respectively for the two cases. It is important to note that survival probabilities for $J / \psi$ and $\Upsilon$ significantly reduce even when the temperature remains smaller than $T_{D}$ for the respective case. The overlap of $\Upsilon(2 S)$ wave function at $T=200 \mathrm{MeV}$ and $\Upsilon(1 S)$ at $T=0$ (Fig.3) gives the transition probability of an initial $\Upsilon$ to the excited state to be about $10 \%$.

We point out the main difference between our approach and the conventional approaches for calculating heavy quarkonium suppression in QGP. In conventional approach, quarkonium suppression is calculated for a QGP medium which has achieved high enough temperature $T_{D}$ so that Debye screening becomes effective in making the quarkonium unbound. If temperature remains below $T_{D}$ one does not expect any suppression of the corresponding quarkonium state. Our approach 


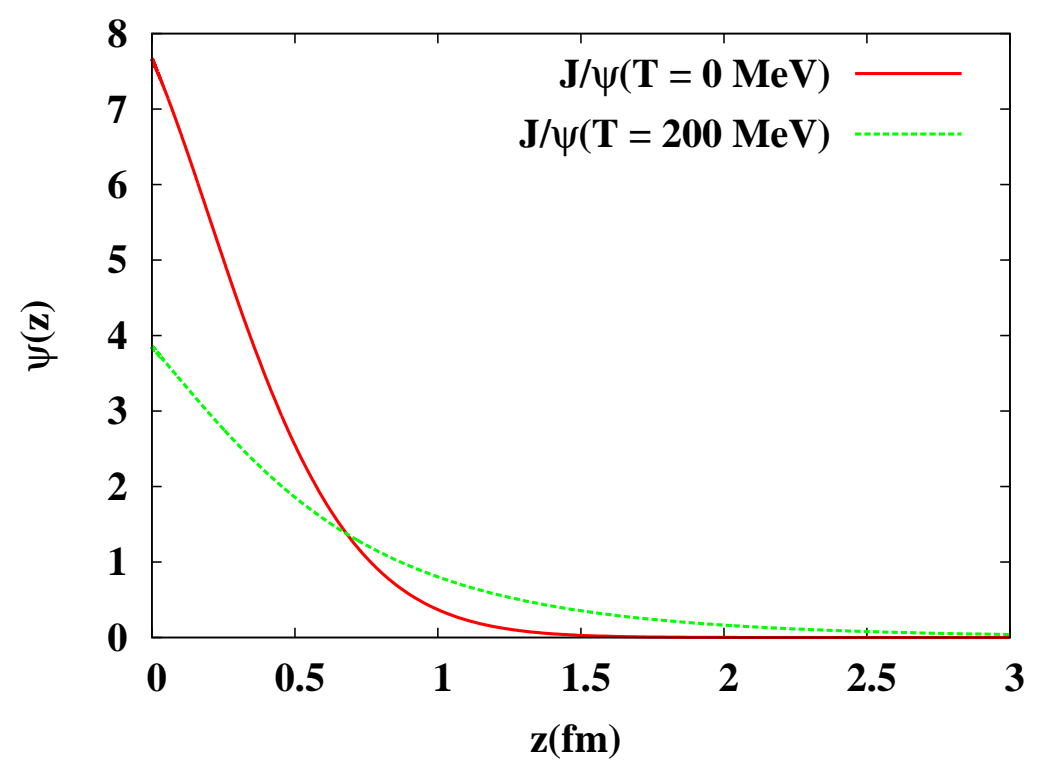

Figure 1: Wave functions for $J / \psi$ states at different temperatures.

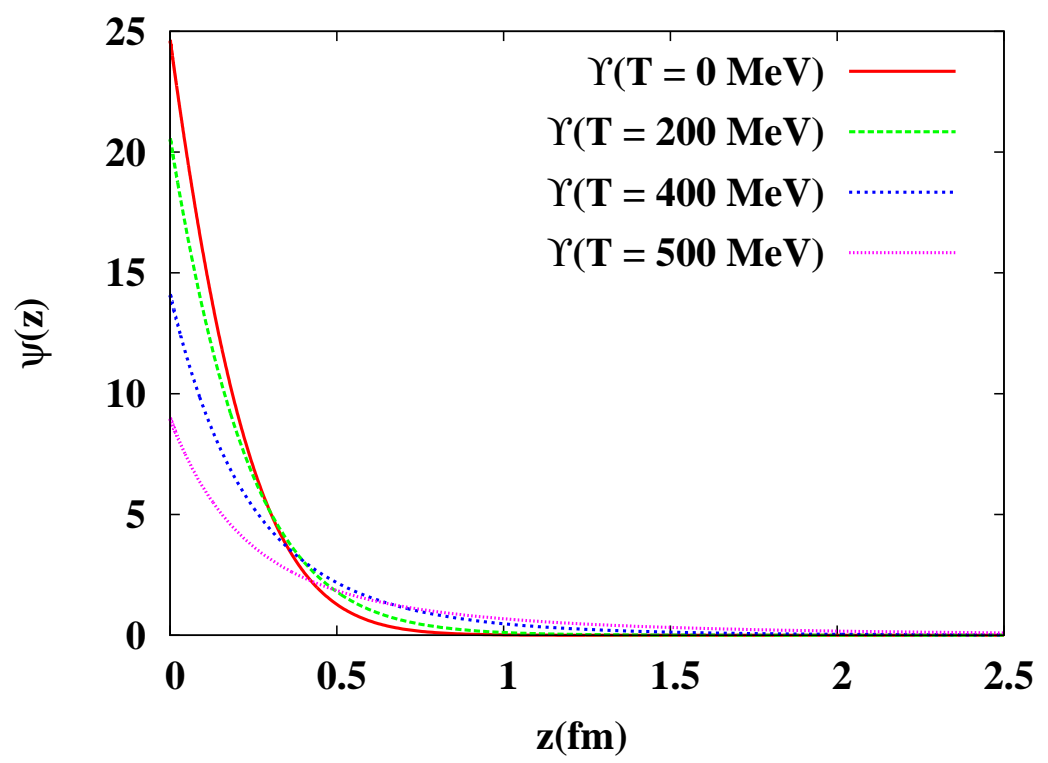

Figure 2: Wave functions for $\Upsilon(1 S)$ states at different temperatures. 


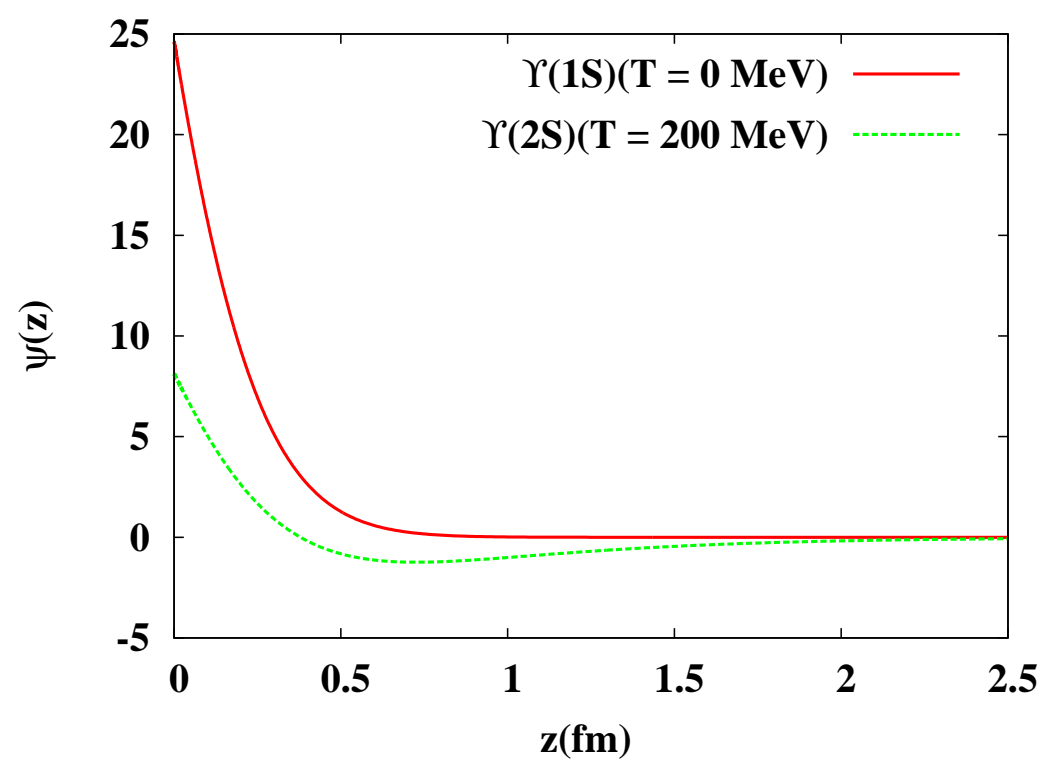

Figure 3: Wave functions for $\Upsilon(1 S)$ and $\Upsilon(2 S)$ states at $T=0$ and $T=200 \mathrm{MeV}$ respectively.

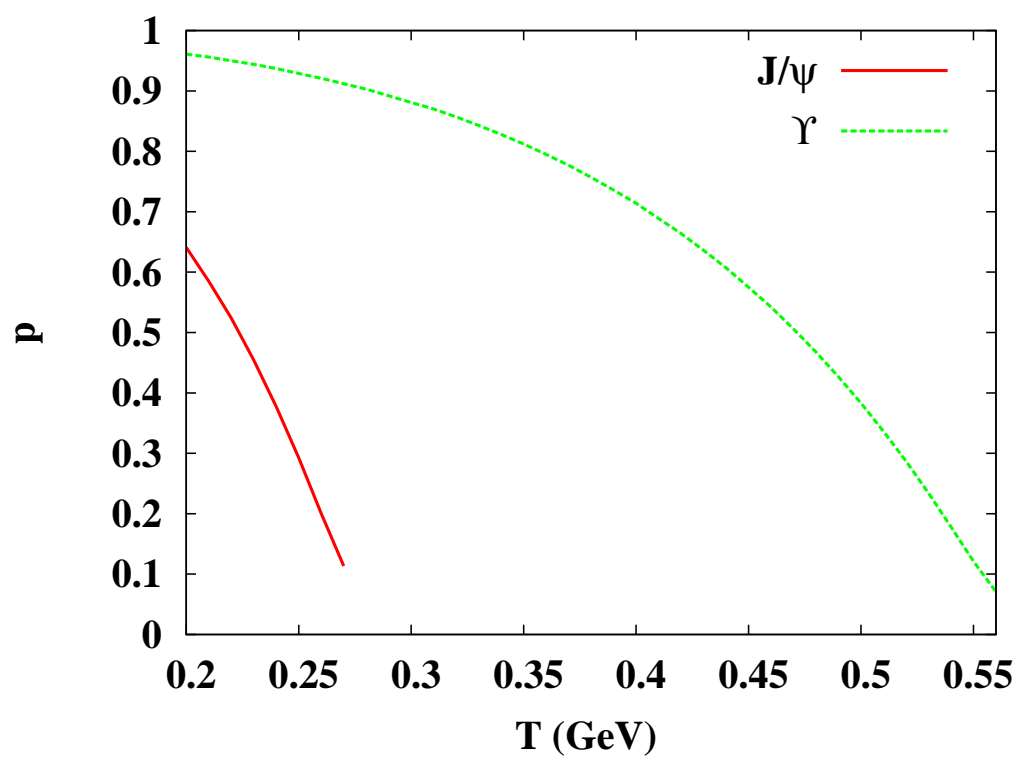

Figure 4: Survival probabilities of initial $T=0 \mathrm{~J} / \psi$ and $\Upsilon$ states in QGP at different temperatures calculated in the sudden (quench) approximation. 
is to focus on the situation when temperature remains below $T_{D}$ (for the specific quarkonium under consideration). If the initial thermalization of QGP happens very slowly in time scale much larger than the time scale of quarkonium which is of order $1 \mathrm{fm}$, then indeed we will conclude that no quarkonium suppression will be expected. However, in ultra-relativistic heavy-ion collisions thermalization is definitely achieved within a time scale of about $1 \mathrm{fm}$ (from elliptic flow measurements) [4], which is of same order as the dynamical scale of $q \bar{q}$ in the quarkonium bound state (or the time scale of transition between relevant states). In such a situation one cannot assume that the initial zero temperature quarkonium state will simply evolve to the finite temperature quarkonium state. Instead, time dependent perturbation theory should be used to calculate the survival probability of the initial quarkonium state. In fact expected thermalization time scale at RHIC and LHC may be as short as $0.25-0.1 \mathrm{fm}$ respectively [3]. With such rapid thermalization, use of sudden perturbation approximation may be appropriate. We calculate survival probability of quarkonium (and transition to excited state for $\Upsilon$ ) and show that even when temperature of QGP remains much below $T_{D}$, the quarkonium state can decay with significant probability. Even if the temperature exceeds $T_{D}$, during initial stages of heating the decay of initial quarkonium state due to time dependent potential, as discussed here, should be incorporated in calculating the final net quarkonium suppression.

One way to clearly distinguish our mechanism from the conventional mechanism is to study quarkonium suppression for varying QGP temperatures and the thermalization time scale independently. One may achieve this by considering different centrality, or rapidity, or by using different combinations of nucleus size and collision energies so that the thermalization time and QGP temperature can be varied independently.

\section{Acknowledgment}

I'm very thankful for the financial support through the ExtreMe Matter Institute (EMMI). We are very thankful to Abhishek Atreya, Arpan Das, Shreyansh S. Dave,Biswanath Layek, and Ananta P. Mishra for useful discussions.

\section{References}

[1] T. Matsui and H. Satz, Phys. Lett. B178, 416 (1986).

[2] M. Abreu et al., NA50 Collaboration, Phys. Lett. B477, 28 (2000); H. Satz, J. Phys. G 32, R25 (2006).

[3] D.M. Elliott and D.H. Rischke, Nucl. Phys. A 671, 583 (2000).

[4] P.F. Kolb and U. W. Heinz, in Quark gluon plasma, page 634, Editor R.C. Hwa (2003); arXiv: nucl-th/0305084.

[5] N. Dutta and N. Borghini, arXiv:1206.2149; N. Dutta, A.K. Chaudhuri, and P.K. Panigrahi, arXiv:1311.2875.

[6] H. Satz, J. Phys. Conf. Ser. 455, 012045 (2013). 